NOTULES HYDRAULIQUES HYDRAULIC BRIEFS

\title{
La schématisation par un ellipsoïde d'une particule sédimentant dans l'eau
}

\author{
The ellipsoïdal schematisation \\ of a particle falling in water
}

\begin{abstract}
Chute des corps en régime de Stokes. Contrairement a l'opinion généralement admise, les corps peuvent tourner en tombant. Ceux qui no tournent pas sont entièrement définis au point de vue de leur chute par 6 coefficients formant matrice symétrique. En général, ils font « feuille morte » en tombant.
\end{abstract}

La chute des corps dans l'eau intéresse de nombreux techniciens. Beaucoup de recherches théoriques et expérimentales ont été faites pour déterminer les lois de ce phénomène. En géné. ral on admet que les corps peuvent ètre schématisés par des ellipsoïdes dont la forme se rapproche le plus possible de celle du corps envisagé.

A la suite de l'article de M. McNown dans un récent numéro de la Houille Blanche (nº 5,1951 ), nous nous proposons d'examiner la valeur de cette schématisation quand la chute dans l'eau se fait en régime de STrokes (c'est-à-dire si la particule est suffisamment petite et la vitesse de chute suffisamment lente pour que les équations simplifiées de l'hydraulique selon STokEs soient applicables ${ }^{1}$ ).

1. Pour le sable ceci a lieu si « le diamètre moyen 》 du grain est inférien à $1 / 10$ de mm.

\begin{abstract}
Fall of parlicles in Stokes' regime. Contraty to general opinion, the particles may revolve as they fall. The fall of those lhat do not revolve is fully defined by six coefficients forming a symmetrical matrix. Particles usually fall lilie a dead leaf.
\end{abstract}

Dans ce cas on peut aborder le problème par le calcul et, pour certains corps de forme simple, déterminer entièrement les lois de la chute. De nombreuses expériences ont d'ailleurs confirmé ces calculs.

La plupart des expérimentateurs ont conclu, à la suite de leurs essais, qu'un corps tombant dans l'eau, en suivant la loi de STokss, ne tourne pas sur lui-mème quelle que soit sa position. (Voir par exemple : Houille Blanche, n" 5 de 1951 , p. 716, 2 colonne, lignes $6,7,8$.)

De même, les mathématiciens qui ont étudié théoriquement l'écoulement visqueux parallèle perturbé par un ellipsoïde ont trouvé que le système des forces hydrauliques appliqué à celui-ci se réduit à une force unique passant par le centre de l'ellipsoíde.

En réalité, tous les essais ont été effectués avec des corps homogènes ayant trois plans de symé- 
trie orthogonaux (ou présentant approximativement une telle symétrie - voir par exemple Trans. Am. Géo. Union 1950, page 77, figure 2). Malgré la grande varićté de forme de ces corps, ces essais ne peuvent nous renseigner que sur un cas très particulier de la chute des corps dans l'eau : celui des corps qui ne peuvent pas tourner en tombant, comme nous le montrerons plus bas; beaucoup de corps dans leur chute tournent sur eux-mêmes. On voit donc à quel point il faut se méfier des généralisations, même après avoir fait un grand nombre d'essais.

Les auteurs de ces essais constatent également que les vitesses de chute des particules sont bien celles auxquelles il faut s'attendre, compte tenu de la schématisation par un ellipsoïde de forme voisine à la leur. Cette dernière constatation ne peut pas non plus être généralisée. En eflet, si une particule tourne en tombant, le mouvement de rotation dissipe aussi de l'énergie; il est done probable que deux particules de forme voisine, dont l'une tourne en tombant, tandis que l'autre ne tourne pas, n'ont pas la même vitesse de chute.

Par contre, les corps qui ne tournent pas, quelle que soit leur orientation, en tombant dans l'eau, peuvent toujours être schématisés par un ellipsoïde et ceci à cause d'un théorème de réciprocité, comme on verra plus loin. Les lois de chute de ces corps sont entièrement définies par trois mouvements de chute suivant trois directions arbitraires.

\section{ÉTUDE DE LA CHUTE DES CORPS EN RÉGIME DE STOKES ${ }^{1}$}

Nous appelons $\mathrm{E}_{1}$ l'écoulement de l'eau autour d'un corps qui tombe en régime établi. Le corps aura alors une vitesse caractérisée par le vecteur $\vec{V}$ et une rotation autour de son centre de gravité caractérisée par $\vec{\Omega}$. Nous appellerons $E_{2}$ un autre écoulement, pour lequel le fluide possède à l'infini la vitesse $-\vec{V}$ et la rotation $-\vec{\Omega}$, et qui est perturbé, à distance finie, par le corps envisagé. Les forces hydrauliques agissant sur la particule sont les mêmes dans ces deux écoulements.

Nous allons montrer qu'un corps homogène ayant trois plans de symétrie orthogonaux ne peut pas tourner en tombant et que toutes ses orientations sont stables. S'il en est ainsi, $\vec{\Omega}=0, \vec{V}$ est indépendant du temps $\overrightarrow{[\vec{V}}(t)$ pourrait etre une fonction périodique]. L'écoulement $E_{2}$ peut être

1. Par la suite, il s'agina toujours de ce cas. décomposé en trois écoulements $\mathrm{E}_{21}, \mathrm{E}_{22}, \mathrm{E}_{23}$ définis par les vecteurs $\vec{V}_{1}, \vec{V}_{2}, \vec{V}_{3}$, projections de $\vec{V}$ sur les intersections des plans de symétrie du corps. La superposition de $E_{21}, E_{22}, F_{23}$ redonne $\mathrm{E}_{2}$, puisque les équations sont linéaires. Les écoulements $\mathrm{E}_{21}, \mathrm{E}_{22}$ et $\mathrm{E}_{23}$ produisent sur le corps des forces hydrauliques qui, par symétrie, sont équivalentes à une résultante générale unique passant par le centre de figure. Comme la superposition s'applique également aux forces hydrauliques, le corps est toujours soumis à une force unique passant par le centre de figure quel que soit l'écoulement $\mathbf{E}_{2}$. Done il existe pour chaque orientation de la particule un écoulement $\mathbf{E}_{1}$ avec une vitesse $\vec{V}$ juste suffisante pour que la résultante générale hydraulique contrebalance le poids. Comme les équations sont linéaires, ces écoulements sont les seuls possibles.

On en conclut que :

les corps homogènes ayant trois plans de symétrie orthogonaux tombent sans tourner, quelle que soit leur orientation.

On peut faire des raisonnements analogues quand les corps ont d'autres éléments de symétrie (axe de symétrie, axe de révolution, etc...). On trouve, selon le cas, un nombre fini ou infini d'orientations pour lesquelles le corps ne peut, à priori, pas tourner en tombant.

Considérons maintenant d'une façon plus générale une particule qui ne tourne pas en tombant dans l'eau et soumettons-la à trois écoulements $\mathrm{E}_{2 i}$ dont les vecteurs $\vec{V}_{i}$ correspondants sont quelconques et ont la grandeur 1. Les résultantes générales des forces hydrauliques correspondantes sont alors trois vecteurs caractérisés par leurs composantes $\dot{\mathrm{T}}_{i j}$.

Si nous soumettons la particule à un autre écoulement $\mathbf{E}_{2}$ caractérisé par $\vec{V}^{\prime}$ de grandeur 1 ) dont les projections sur les vecteurs $\vec{V}_{i}$ sont $\alpha_{i}$, la nouvelle résultante générale des forces hydrauliques aura les projections :

$$
\sum_{i} \mathrm{~T}_{i j} \alpha_{i}
$$

Nous voyoms que toute les forces hydrauliques sont déterminées par le tenseur $\mathrm{T}_{i j}$. Or, ce tenseur est symétrique, comme le montre le théorème analogue à celui de BrTtr en élasticité.

Par conséquent, toutes les forces hydrauliques peuvent être représentées par un ellipsö̈de. Ceci est d'ailleurs toujours vrai pour les écoulements $E_{2}$, même si le corps tourne en tombant. 
On en conclut pratiquement que:

pour les corps qui ne tournent pas en tombant, il existe trois orientations orthogonales 2 à 2 , pour lesquelles le corps tombe verticalement. Pour toutes les autres orientations, il fait "feuille morte".

Soient $V_{1}, V_{2}$ et $V_{3}$ les vitesses de chute correspondant à ces trois directions privilégiées 1 ). On peut facilement calculer la vitesse de chute pour une orientation quelconque caractérisće par les projections $\beta_{1}, \beta_{2}, \beta_{3}$ de la verticale sur les directions D.

La vitesse de la particule par rapport au fluide est :

$$
V=\sqrt{\beta_{1}{ }^{2} V_{1}{ }^{2}+\beta_{2}{ }^{2}} V_{22^{2}+\beta_{3}{ }^{2} V_{3}{ }^{2}}
$$

Ia projection de cette vitesse sur la verticale (c'est-à-dire la vaic vitesse de chute) est :

$$
v=\beta_{1}^{2} V_{1}+\beta_{2}^{2} V_{2}+\beta_{3}{ }^{2} V_{3}
$$

L'angle 0 de glissement en «feuille morte » est donné par $\cos \theta=\frac{v}{V}$.

Les grandeurs $V$ et $v$ sont aussi données par deux ellipsoïdes lies, comme on sait, au tenseur mentionné plus haut.

Le maximum de l'angle $\theta$ se voit le mieux sur le graphique de Morn lié an tensem (fig. 1).

$$
\cos \theta_{0}=\frac{2 \sqrt{V_{1} V_{3}}}{V_{1}+V_{3}}
$$

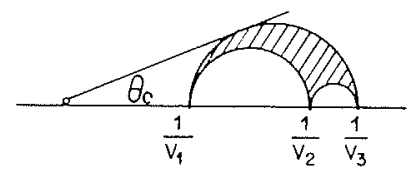

Fig. 1 .

Montrons maintenant quil existe par contre des corps qui ne sont pas stables, quelle que soit Icur orientation.

D'abord, il faut remarquer que, jusqu'ici, seuls des corps ayant trois plans de symétrie orthogonaux ont été abordés par le calcul. Néanmoins, nous avons pu étudier un corps n'ayant que deux plans de symétrie. On montre facilement, à priori, que les forces hydrauliques se réduisent encore par symétrie à une résultante générale sans couple. Mais, dans le cas étudié, cette résultante ne passe pas par le centre de gravité. Io corps ne peut avoir que deux orientations pour lesquelles il ne tourne pas et, il n'y en a qu'unc qui est stable. Le corps étudié était un cylindre infini dont la base est représenté sur la figure?.

On a done prouvé qu'il existait des corps homogènes qui, en tombant, sont soumis à des for-

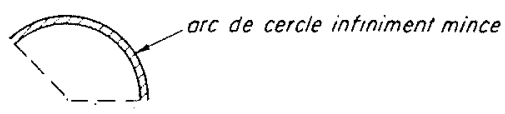

FIG. 2.

ces hydrauliques dont la résultante ne passe pas par le centre de gravité. Nous espérons pouvoir publier ultérieurement les calculs complets.

En ce qui concerne les corps non homogenes, il est évident que la résultante ne passe pas toujours par le centre de gravité et que, en général, ils n'ont pas plus d'une orientation stable. On peut se demander si les forees hydrauliques peuvent comporter un couple. Si ceci est le cas, aucune orientation du corps ne serait stable. Les corps seront alors encore moins symétriques et nous ne connaissons pas d'ctudes faites sur ce sujet jusqu’à présent.

Nóanmoins, on peut « construire 》 un corps qui soit somis à un couple de Ia facon suivante : on considere un écoulement $u \equiv u_{0} \quad \nu \equiv 0 \quad b D \equiv 0$ perturbé par des singularités produisant des vitesses tendant vers zéro quand on s'éloigne veis l'infini. Une première série de ces singularites peuvent produire un couple sur une sphère $\mathrm{Z}$ de rayon très grand $\int f(\mathrm{~T} \cdot \overrightarrow{d \sigma}) \wedge \overrightarrow{\mathrm{OM}}=\overrightarrow{\mathrm{r}}$. The deuxiène série de singularités produisant des vitesses décroissant encore plus vite que les promières peut ne plus avoir d'action sur $\Sigma$, mais permet d'avoir une surface $S$ à distance finie sur laquelle $u \equiv v \equiv w \equiv 0$. Les singularités peuvent ètre distribuées dans une partie du volume délimité par S. S est alors la particule cherchée. Le liquide entre 2 et $s$ transmet le couple $\vec{\Gamma}$ à la particule.

R. MEYER,

Ingénient an Laboratoire Damphinois d'Hydranlique (Nerpie-Grenoble). 